\title{
Determination of candidates for adjunctive 'gastroprotective' prostaglandins
}

\author{
RAYMOND A. ADELIZZI, DO
}

Long-term use of nonsteroidal anti-inflammatory drugs (NSAIDs) is now recognized as being frequently associated with asymptomatic gastric ulceration and its complications. Such adverse effects on the gastric mucosa have recently been found to occur with even greater frequency in certain NSAID-treated patients. Synthetic prostaglandin analogues have been shown to be cytoprotective of the gastric mucosa. This benefit has been evidenced by the reduced risk of gastric ulceration and gastric bleeding in patients taking NSAIDs on a prolonged basis. The first synthetic prostaglandin to be approved for this indication is misoprostol (Cytotec).

To determine which and how many patients receiving NSAIDs should be treated adjunctively with a cytoprotective prostaglandin to reduce risk of NSAIDinduced gastropathy, a clinical stùdy of 100 consecutive patients seen in a rheumatology practice was undertaken. $\mathrm{Pa}$ tients were assessed by interview and chart review. Of these patients, $19 \%$ to $30 \%$ were considered potential candidates for adjunctive prostaglandin therapy. The rationale for use of the "gastroprotective"

From the University of Medicine and Dentistry of New Jersey-School of Osteopathic Medicine, Stratford. Dr Adelizzi is associate professor of medicine and chief of the Division of Rheumatology, Department of Internal Medicine.

Reprint requests to Raymond A. Adelizzi, DO, 301 South Central Plaza, Suite 3100, Stratford, NJ 080841504. prostaglandins appears to lie in the identification of those patients who are especially at high risk for gastric ulcers.

Salicylates and the other nonsteroidal antiinflammatory drugs (NSAIDs) are the most common class of antiarthritic agents prescribed, with more than 67 million prescriptions written each year in the United States. The NSAIDs make up a market that exceeds $\$ 2$ billion. ${ }^{1}$

The widespread use of NSAIDs is due to their clinical effectiveness. There are several postulated mechanisms of action for NSAIDs, with the classical hypothesis being that of prostaglandin synthesis (via inhibition of the enzyme cyclo-oxygenase). ${ }^{2}$

Most of the adverse effects of the NSAIDs are, likewise, due to the inhibition of prostaglandin synthesis and include NSAID-induced gastropathy (ie, gastric erosions, gastric ulcers, and accompanying upper gastrointestinal [GI] tract bleeding). ${ }^{3}$

Prostaglandins of the E series (PGEs) are essential to cytoprotection of the gastric mucosa because they inhibit gastric acid secretion, stimulate gastric mucous and bicarbonate production, and regulate mucosal blood flow. ${ }^{4}$ Knowledge of these mechanisms of action has provided a good rationale for the development of synthetic prostaglandin analogues and their adjunctive use with NSAIDs in selected patients at high risk for gastriculcers.

Research studies of several prostaglandin analogues have been conducted and their find- 
ings published . $^{3,5-8}$ Misoprostol (Cytotec) is the first of the oral "gastroprotective" (cytoprotective) prostaglandins to be approved for use in preventing NSAID-induced gastric ulcers and concomitant GI blood loss. Studies by Graham and coworkers ${ }^{4}$ and others ${ }^{9-13}$ have shown the clinical efficacy of misoprostol for this indication.

Taking into account some of the known risk factors for NSAID-induced gastropathy, Fries and coworkers ${ }^{14}$ studied patients being treated at a rheumatology outpatient office to determine the incidence of potential candidates for adjunctive therapy with an oral gastroprotective prostaglandin.

\section{Patients and methods}

One hundred consecutive patients treated at a university rheumatology outpatient office during January and February 1989 were assessed via an interview and a chart review to identify those who currently used NSAIDs and those who were at high risk for NSAID-induced gastropathy. High-risk subgroups were defined as (1) patients with a previous GI intolerance to NSAIDs, (2) those with dyspepsia related to current NSAIDs therapy, (3) those with a history of peptic ulcer or upper GI tract bleeding, and (4) those more than 65 years old during current NSAID therapy. The study population largely comprised long-standing patients of the investigator, many of whom he had personally followed up for several years.

Assessments of the patients revealed various rheumatologic diagnoses in both the population that was receiving NSAIDs and the one that was not. The most common definitive diagnoses were osteoarthritis, systemic lupus erythematosus, and rheumatoid arthritis (Table). All of the patients had rheumatologic complaints either at the time of the study or during previous visits.

The average age of the entire study group was 55.1 years, with patients who were currently receiving NSAIDs being slightly older (56.4 years) than those who were not (54.2 years). There was a 3:1 female preponderance.

\section{Results}

Of the 100 consecutive patients evaluated, 42
Table

Primary Rheumatologic Diagnoses of
Study Population*

\begin{tabular}{|lcc|}
\hline Diagnosis & $\begin{array}{c}\text { No. of } \\
\text { patients } \\
\text { taking } \\
\text { NSAIDs }\end{array}$ & $\begin{array}{c}\text { No. of } \\
\text { patients } \\
\text { not taking } \\
\text { NSAIDs }\end{array}$ \\
\hline Osteoarthritis & 11 & 10 \\
Rheumatoid arthritis & 10 & 5 \\
Juvenile rheumatoid arthritis & 1 & \\
Systemic lupus erythematosus & 5 & 19 \\
Mixed connective tissue disease & 1 & \\
Bursitis/tendinitis & 4 & 2 \\
Arthralgia of undetermined & & \\
$\quad$ origin & 6 & 3 \\
Carpal tunnel syndrome & 1 & 2 \\
Osteoporosis & 1 & 5 \\
Psoriatic arthritis & 1 & 1 \\
Arthropathy of inflammatory & & \\
$\quad$ bowel disease & 1 & 2 \\
Scleroderma & & 1 \\
Pseudogout & & 1 \\
Gout & & 2 \\
Polyarteritis nodosa & & 1 \\
Hypersensitivity vasculitis & & 1 \\
Polymyalgia rheumatica & & 1 \\
Temporal arteritis & & 1 \\
Erythema nodosum & & 1 \\
Purpura of undetermined origin \\
Total & & $\mathbf{4 2}$ \\
\hline *Based on evaluations of 100 consecutive patients. & \\
\hline
\end{tabular}

were taking NSAIDs at the time of the study. Of these, $19(45 \%)$ were categorized into one of the four previously defined high-risk subgroups for NSAID-induced gastropathy (Fig 1). Additionally, $11(11 \%)$ other patients of the total study group were not taking NSAIDs at the time; however, they could have been considered to be at high risk and therefore candidates for cytoprotective prostaglandins (Fig 2).

When analyzed together, the patients from the two main high-risk groups represented $30 \%$ of the total study population who were candidates for adjunctive prostaglandin therapy. When patients with current or previous NSAID-induced GI intolerance were excluded, and only those with a history of peptic ulcer/ upper GI tract bleeding or those older than 65 years were considered, then the percentage of the total study population who were candidates for adjunctive prostaglandin therapy was $23 \%$. 
Figure 1. Patients taking nonsteroidal anti-inflammatory drugs (NSAIDs) at high risk for NSAIDinduced gastropathy. (Note: Some patients may have been categorized in more than one high-risk subgroup.)

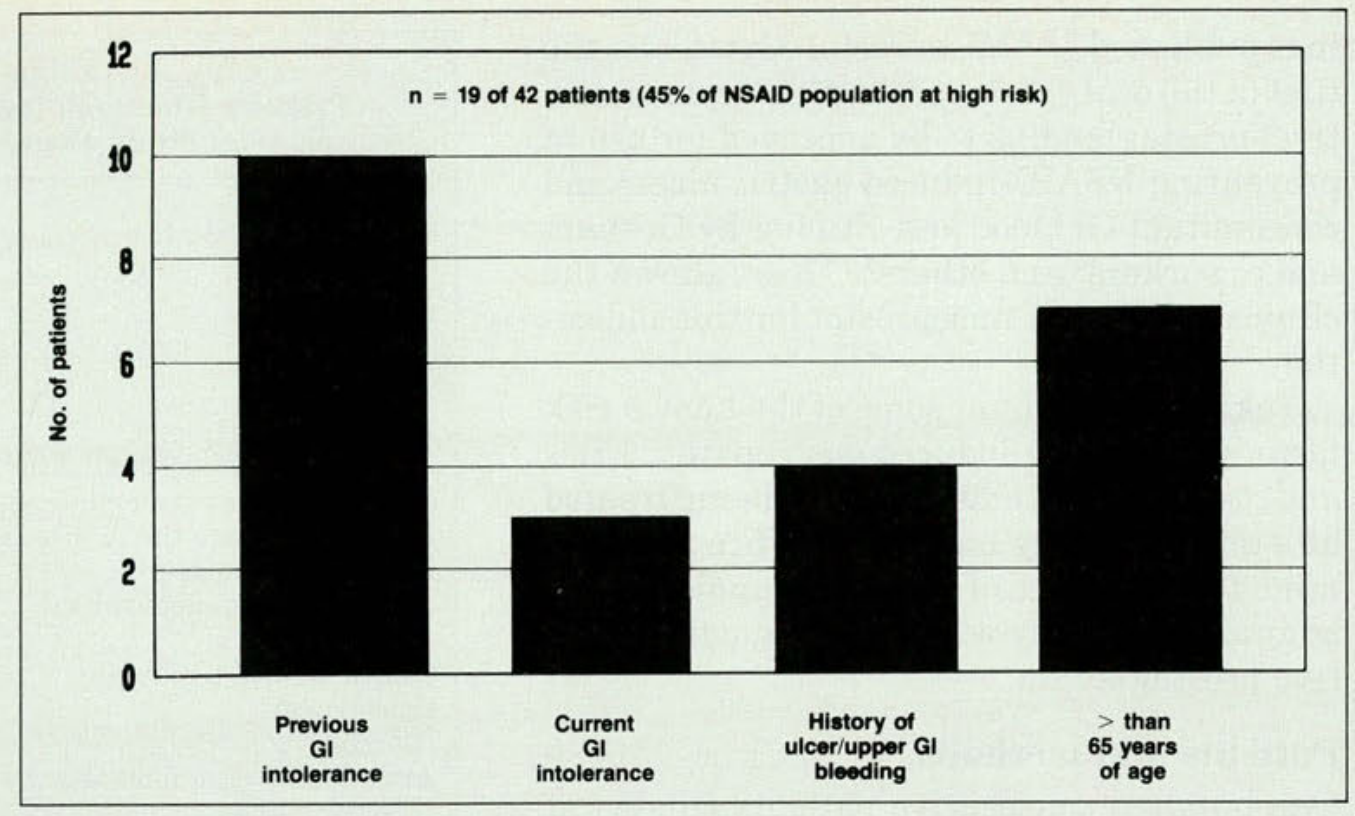

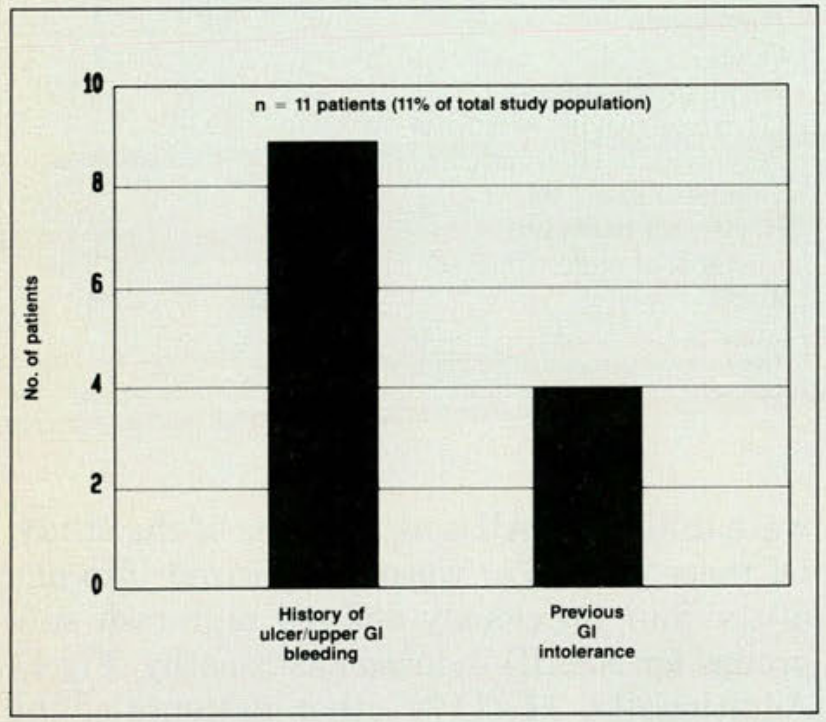

Figure 2. Patients not taking nonsteroidal anti-inflammatory drugs (NSAIDs) who also were possible candidates for gastroprotective prostaglandins. (Note: Some patients may have been categorized in both high-risk subgroups.)

\section{Discussion}

According to data provided by the American Rheumatism Association Medical Information System (ARAMIS), an estimated 2500 deaths at the least occur annually from NSAID-associated GI tract bleeding. ${ }^{1,14}$ In patients taking NSAIDs, mortality associated with peptic ulcer complications has been shown to be more than double that in patients not taking NSAIDs. ${ }^{15}$ Also, NSAIDs are taken twice as often by patients older than 60 years found to have bleeding ulcers as compared with an age-matched community control or hospital inpatient control population. ${ }^{16}$

Various endoscopy studies have shown the incidence of peptic ulcers in patients taking NSAIDs to be approximately $20 \% .^{1,4,17}$ Generally, a poor correlation has been found between complaints of GI symptoms and endoscopic confirmation of pathoses. ${ }^{17,18}$ Another problem related to NSAID-induced gastropathy has been recognized relatively recently: in patients taking NSAIDs, ulcers and other significant endoscopic lesions typically occur asymptomatically. Larkai and coworkers ${ }^{18}$ found that more than $90 \%$ of the patients who had endoscopic gastric lesions while taking NSAIDs were asymptomatic. This finding may be attributed to the anti-inflammatory/analgesic properties of the NSAIDs themselves.

The concept of NSAID-related gastropathy and the identification of those patients particularly at risk were succinctly reviewed in 1988 by Roth, ${ }^{1}$ and more recently by Fries and coworkers. ${ }^{14}$ Certainly patients who are elderly or who have a history of gastric ulcers or gastric bleeding should be considered to be at significant risk. According to Fries and coworkers, ${ }^{14}$ there is an increased risk of NSAIDinduced gastropathy also in patients who are taking corticosteroids; those who have been diagnosed as having rheumatoid arthritis; those 
who have had upper abdominal pain in the past 6 months; those who have discontinued any NSAID because of adverse GI effects; those who have taken antacids, H-receptor antagonists, or sucralfate in the past 6 months; and those who smoke cigarettes.

Most NSAIDs (except perhaps the nonacetylated salicylates) alter the cytoprotective mechanisms of the stomach by reducing endogenous prostaglandin production. Misoprostol, a prostaglandin $\mathrm{E}_{1}$ analogue, has been shown to effectively prevent gastric ulcers in patients taking aspirin as well as in patients taking other NSAIDs. ${ }^{8-13}$ The drug may also help alleviate dyspepsia or abdominal pain associated with NSAIDs, but this benefit has not been adequately or consistently demonstrated. ${ }^{4,5}$ Therefore, it remains debatable whether to consider patients experiencing these side effects as potential candidates for adjunctive prostaglandin therapy.

An important question, then, for the physician prescribing NSAIDs is, Which patients should receive adjunctive therapy with a gastroprotective prostaglandin? In view of the favorable safety profile of misoprostol (administration to pregnant women excluded), it would seem beneficial to prescribe this drug for those patients at high risk for NSAID-related gastropathy, especially elderly patients or patients with a history of peptic ulcers or upper GI tract bleeding. In the current study, these made up $23 \%$ of the patient population.

It must be noted that although approximately $19 \%$ to $30 \%$ of the patients in the current study were potential candidates for a cytoprotective prostaglandin as an adjunct to arthritis therapy, these percentages may be conservative. Not all the risk factors as delineated by Roth ${ }^{1}$ and Fries and coworkers ${ }^{14}$ were considered (eg, patients who took corticosteroids, smoked cigarettes, etc). Also, although it is believed that the majority of the current study population had taken NSAIDs at one time or another (this was not specifically evaluated), only $42 \%$ were taking NSAIDs at the time of the study. This percentage may have already reflected, in part, the curtailed use of NSAIDs by the elderly and by those with a history of peptic ulcers.

In conclusion, misoprostol, and eventually other cytoprotective prostaglandins, should be considered-especially for those patients at high risk-to reduce the morbidity and mortality associated with NSAID-induced gastric complications.

1. Roth SH: NSAID and gastropathy: A rheumatologist's review. J Rheumatol 1988;15:912-919.

2. Schlegel SI, Paulus HE: Update on NSAID use in rheumatic diseases. Bull Rheum Dis 1986;36(6):1-8.

3. Aly A: Prostaglandins in clinical treatment of gastroduodenal mucosal lesions: A review. Scand $J$ Gastroenterol 1987;22(suppl 137):43-49.

4. Graham DY, Agrawal NM, Roth SH: Prevention of NSAIDinduced gastric ulcer with misoprostol: Multicentre, doubleblind, placebo-controlled trial. Lancet 1988;2:1277-1280.

5. McColl KEL: Assessment of two new therapies for peptic ulcer disease: Omeprazole and the prostaglandin analogues. Dig Dis 1988;6:65-75.

6. Inauen W, Wyss PA, Kayser S, et al: Influence of prostaglandins, omeprazole, and indomethacin on healing of experimen-

al gastric ulcers in the rat. Gastroenterology 1988;95:636-641.

7. Dammann HG, Dreyer M, Walter THA, et al: Rioprostil 600 $\mu \mathrm{g}$ nocte: High duodenal ulcer healing rates, low incidence of side effects. Prostaglandins in Clinical Research 1987, pp 289-293.

8. Goa KL, Monk JP: Enprostil-A preliminary review of its pharmacodynamic and pharmacokinetic properties, and therapeutic efficacy in the treatment of peptic ulcer disease. Drugs 1987;34:539-559.

9. Lanza F, Peace K, Gustitus L, et al: A blinded endoscopic comparative study of misoprostol versus sucralfate and placebo in the prevention of aspirin-induced gastric and duodenal ulceration. Am J Gastroenterol 1988;83:143-146.

10. Silverstein FE, Kimmey MB, Saunders DR, et al: Gastric protection by misoprostol against $1300 \mathrm{mg}$ of aspirin: An endoscopic study. Dig Dis Sci 1986;31(February suppl):137S-141S. 11. Ryan JR, Vargas R, Clay GA, et al: Role of misoprostol in reducing aspirin-induced gastrointestinal blood loss in arthritic patients. Am J Med 1987;83(suppl 1A):41-44.

12. Aadland E, Fausa O, Vatn M, et al: Protection by misoprostol against naproxen-induced gastric mucosal damage. Am $J$ Med 1987;83(suppl 1A):37-40.

13. Lanza FL, Aspinall RL, Swabb EA, et al: Double-blind, placebo-controlled endoscopic comparison of the mucosal protective effects of misoprostol versus cimetidine on tolemetin-induced mucosal injury to the stomach and duodenum. Gastroenterology 1988;95:289-294.

14. Fries JF, Miller SR, Spitz PW, et al: Toward an epidemiology of gastropathy associated with nonsteroidal anti-inflammatory drug use. Gastroenterology 1989;96:647-655.

15. Armstrong CP, Blower AL: Non-steroidal anti-inflammatory drugs and life threatening complications of peptic ulceration. Gut 1987;28:527-532.

16. Somerville K, Faulkner G, Langman M: Non-steroidal antiinflammatory drugs and bleeding peptic ulcer. Lancet 1986;1:462-464.

17. Caruso I, Porro GB: Gastroscopic evaluation of anti-inflammatory agents. $\mathrm{Br} \mathrm{Med} J$ 1980:280:75-78.

18. Larkai EN, Smith JL, Lidsky MD, et al: Gastroduodenal mucosa and dyspeptic symptoms in arthritic patients during chronic nonsteroidal anti-inflammatory drug use. Am J Gastroenterol 1987;82:1153-1158. 